\title{
Immunomodulatory properties of anticancer
} monoclonal antibodies: is the 'magic bullet' still a

\section{reliable paradigm?}

\author{
"There has been an exponential increase in the development of monoclonal \\ antibodies ... and other target-specific antitumor drugs for the treatment \\ of human cancer over the last 15 years."
}

\section{KEYWORDS: biological response $\approx$ cancer immunity $\approx$ modifier $\approx$ molecular-target therapy $=$ monoclonal antibodies}

There has been an exponential increase in the development of monoclonal antibodies (mAbs) and other target-specific antitumor drugs for the treatment of human cancer over the last 15 years. The encouraging results obtained from large clinical trials have refashioned the 'magic bullet' paradigm. Ehrlich hypothesized the possibility of using antibodies such as killing darts to discriminate cancer cells from their normal counterparts. This paradigm has represented a conceptual alternative to the conventional cytotoxic chemotherapy, which is considered poorly selective for cancer tissue and, therefore, associated with significant toxicity [1,2] .

\section{"...mAbs actively interact with immune system and show significant immune-modulating effects..."}

The magic bullet theory was formulated in the early 20th Century when the knowledge of cancer cell biology and the immune surveillance system was first understood. At present, we believe that the large amount of information accumulated over the years clearly demonstrates that the mechanisms of anticancer activity of these agents is much more complex owing to the fact that mAbs actively interact with the immune system and show significant immunemodulating effects, largely contributing to the final antitumor activity in patients. In addition, poor multidisciplinary interaction in the research methodology aimed at assessing the activity of anticancer biological agents has pushed researchers to investigate anticancer mAbs as molecular target inhibitors and not as 'old-fashioned' biological immune agents. This approach has also been enforced by the fact that only mAbs directed at functional molecular structures such as EGF receptor (EGFR), IGF receptor,
HER2/neu, VEGF and CD20 have shown significant antitumor activity $[3,4]$. On the basis of this, when these hypothetic magic bullets were exploited in clinical translation, any potential issue concerning their immunological nature or interference with the host microenvironment has been considered superfluous and, consequently, poorly investigated. Recently, the research concerning these agents has been addressed owing to the identification of further biochemical targets critical for tumor cell growth and survival and, eventually, to the potential synergistic interaction with conventional anticancer cytotoxic drugs.

At present, there is no direct evidence demonstrating that the antitumor activity of the majority of $\mathrm{mAbs}$ used in clinical practice is related to the inhibition of specific target transducers. In addition, in many cases, there is not even a clear demonstration that the expression of $\mathrm{mAb}$ targets on the cancer cells and in the tumor area is predictive of response to the treatment (i.e., EGFR and cetuximab/panitumumab, or VEGF/VEGF receptor and bevacizumab) [5]. Furthermore, it has been shown that the use of alternative small molecules that have the ability to inhibit the same pharmacological targets as mAbs (e.g., erlotinib in place of cetuximab for EGFR inhibition in colon cancer) and provide a much stronger biochemical activity in vitro does not produce any anticancer effect in the clinical setting [6].

Many hypotheses and theories have been formulated in support of the current vision of $\mathrm{mAb}$ activity; however, it is clear that the use of $\mathrm{mAbs}$ as molecular target inhibitors, in accordance with the magic bullet paradigm, is considered simply to be a scientific dogma. Clear examples are represented by $\mathrm{mAbs}$ raised against EGFR families, such as cetuximab and panitumumab, which recognize EGFR-1, and

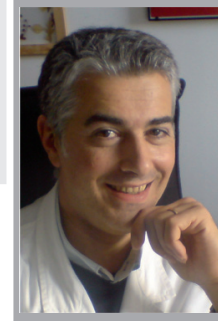

Pierpaolo Correale Author for correspondence: Operative Unit of Medical Oncology Azienda Ospedaliera Universitaria Senese, Istituto Toscano Tumori, Viale Bracci 11, 53100, Siena, Italy correalep@yahoo.it

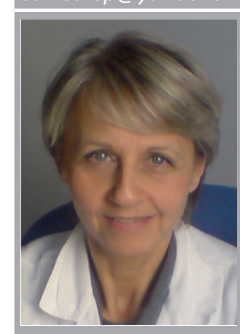

Maria Grazia Cusi

Section of Microbiology, Department of Molecular Biology, Siena University, Viadelle Scotte 5, 53100, Siena, Italy

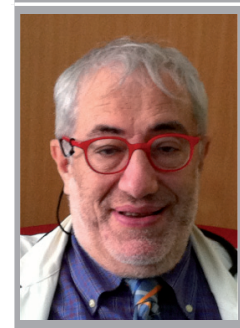

Pierosandro Tagliaferri

Medical Oncology Unit, Campus Salvatore Venuta, Magna Graecia University \& Tommaso Campanella Cancer Center, Loc Germaneto, Catanzaro, Italy 
transtuzumab and pertuzumab, which recognize EGFR-2/HER-neu. The clinical use of these $\mathrm{mAbs}$ is currently based on the knowledge that these $\mathrm{mAbs}$ are able to interfere with receptor dimerization and autophosphorylation, which trigger downstream intracellular cascades and, finally, regulate the expression of genes involved in cell proliferation, apoptosis protection, cell motility and production of neoangiogenic factors [6].

\section{"...other mAbs, such as bevacizumab, that trap free VEGF in the bloodstream, may have direct and powerful immune-mediated effects..."}

It has been hypothesized that the use of these mAbs, by interfering with these mechanisms, may indeed induce a direct antiproliferative effect on the tumor cells, restore the sensitivity to proapoptotic stimuli and sensitivity to cytotoxic drugs and, finally, generate a direct antiangiogenic antimetastatic effect. However, in the last few years, compelling evidence from experimental and clinical research indicates that the effective mechanism of antitumor activity of these $\mathrm{mAbs}$ is indeed related to their immunological properties. The type and timing of tumor response to some of these mAbs, such as cetuximab, rituximab and trastuzumab, and the strong correlation between survival and selected patients' polymorphisms of fragment crystallization region $(\mathrm{Fc})$ receptors $(\mathrm{FcR}) \gamma \mathrm{III}$ a and $\gamma \mathrm{IIa}$, clearly indicate that the antibody-dependent cell-mediated cytotoxicity (ADCC), as well as a consequent T-cell-mediated immunological response, may play a critical role in their final therapeutic effect. The same mechanisms of resistance and escape utilized by cancer cells against these agents appear to be different from that used against other drugs with molecular targets and cytotoxic drugs. A clear example is represented by the knowledge that retreatment with trastuzumab beyond progression after firstline combination therapy still produces clinical benefits when compared with chemotherapy alone $[4,7,8]$.

Morevover, other mAbs, such as bevacizumab, that trap-free VEGF in the bloodstream, may have direct and powerful immunemediated effects, if one takes in consideration the pleiotropic and immune-suppressive role played by this growth factor in stimulating inhibitory myeloid cells, regulatory $\mathrm{T}$ cells and inducing the production of immunosuppressive cytokines such as IL-10 and IL-13 [9,10].
With regard to the recognized clinical efficacy of mAbs, these observations make clear that their antitumor activity is due not only to their ability to bind to specific antigens associated with tumor phenotype, but also to the specific functions mediated by their Fc. The latter may, in fact, mediate complement-dependent cytotoxicity, ADCC and multiple immunological interactions [11-17].

Monoclonal antibodies behave similarly to normal antibodies produced during an ordinary humoral response to nonself antigens. Via the Fc fragments, mAbs in fact communicate with the other branches and effectors of the immune surveillance system in the attempt to provide a coordinated response to the specific antigen source (bacterial or viral infections or occurrence of transformed cells). This type of interaction is not the same across all of the antibodies but it is clearly related to the specific isotype. Isotypespecific Fcs bind with different affinities, with multiple FcRs expressed on the surface of different immune-competent cell lineages leading to different immunological effects.

\section{"Isotype-specific Fcs bind with different affinities, with multiple FcRs expressed on the surface of different immune-competent cell lineages leading to different immunological effects."}

Human $\operatorname{IgG}_{1}$ and $\mathrm{IgG}_{3}$ Fcs, in particular, bind to FcR $\gamma \mathrm{III}$ (expressed by NK cells, macrophages and dendritic cells [DCs]) with a greater affinity than FcR $\gamma \mathrm{II}$ (expressed by DCs and other antigen-presenting cells, as well as by myeloid cells) and FcR $\gamma \mathrm{Ia}$ (granulocytes and myeloid cells). Owing to these features, these classes of mAbs mainly lead to NK cell-mediated ADCC killing of target cells and myeloid cell-mediated phagocytosis. Conversely, human $\mathrm{IgG}_{2} \mathrm{Fc}$ binds FcR $\gamma I I$ Ia and FcR $\gamma$ Ia with greater affinity; thus, they are prone to activating myeloid cell-mediated ADCC killing and opsonization, with consequent antigen cross-priming in place of a NK cell-mediated cytotoxicity. These previously mentioned differences in FCR binding affinity represent a main bioengineering topic in this research field, with attempts to identify specific amino acidic substitutions or glucosidic changes in the Fcs, which are able to enhance the antitumor activity of the mAb, by improving NK-mediated ADCC, phagocytosis and/or antigen cross-presentation [13].

The previously mentioned FcRs (i.e., $\gamma \mathrm{Ia}, \gamma \mathrm{II} \mathrm{a}$ and $\gamma$ IIIa), once engaged by the Fc fragment, are capable of triggering a kinase-dependent 
activating biochemical signal in the immunecompentent cells [13]. The final result would be the activation of:

- NK and NK T cells, which enhance their killing function (large granular cells);

- Granulocytes and macrophages, whose maturation is represented by an increased ability to phagocytate antibody-opsonized cells and to produce lytic enzymes;

- Dendritic cells, which increase the membrane expression of antigen-derived peptide bound class I and II HLA molecules, CD83 and coaccessory molecules such as CD80, CD86 and CD40.

Once activated by the FcR engagement, DCs are able to take up antigens released by cancer cells, promote phagocytosis or trogocytosis and process tumor cell-derived antigens with consequent enhanced antigen presentation in a contest of danger signals antigen-specific T-cell precursors, with final induction of memory and antigen-specific immune effectors [18]. In order to trigger the previously described events, FcRs have to bind to the Fcs of mAbs, which in turn have already bound the target antigen. In this context, the antigen may be free or expressed on the surface of target cells (opsonization). No interaction between $\mathrm{mAbs}$ and $\mathrm{FcRs}$ is possible with free soluble antibodies unbound to target antigens [19].

All these considerations on the immunemodulating properties of $\mathrm{mAbs}$ depict a novel scenario for their use in cancer patients. In fact, when there is an excess of free antibody (different affinity escalating from $\mathrm{IgG}_{2}>\mathrm{IgG}_{1}>\mathrm{IgG}_{3}$ ) in the bloodstream, a powerful feedback inhibitory mechanism is ignited, which induces irreversible immune depression. This effect is mediated by the inhibitory Fc $\chi I I b R$, which is expressed on DCs, macrophages and other myeloid cells. This receptor is able to bind free $\mathrm{mAbs}$ and its engagement activates an intracellular phosphatase, which at first downregulates the activating receptors (Ia and IIa) and subsequently stimulates the production of inhibitory cytokines (e.g., IL-10 and IL-13), stopping any subsequent $\mathrm{T}$ - and B-cell-mediated immune response. This mechanism is physiologically required to stop a specific response when the invading antigen source has been eliminated in order to avoid dangerous overreactions [18]. In this context, an exceeded dosage of mAbs could have a detrimental and prolonged consequence on patient survival and occurrence of adverse events.

Specific efforts should be directed at research into agents and combination strategies that are able to enhance the immunomodulating properties of mAbs. Different isotypes or mutated Fc segments should be investigated by taking into account their biological nature.

A correct integration of $m A b$ therapy with the administration of inflammatory cytokines such as IL-2 and -12 could enhance NK cell interaction with target cells covered by mAbs. Moreover, anticancer drugs such as fluorouracil and gemcitabine administered prior to $\mathrm{mAb}$ administration could induce antigen re-editing (induction of neoantigens and/or upregulation of pre-existing antigens) in cancer cells and activate powerful danger signals (i.e., HSP-90 and calreticuline). Once opsonized and/or orphagocyted by DCs and macrophages, these cells could become a great source of neoantigens available for an efficient antigen-specific T-cell response with long-term memory.

In light of this, we believe that further studies should be designed to investigate mAbs as 'oldfashioned' biological response modifiers in the place of magic bullets.

\section{Financial \& competing interests disclosure}

The authors have no relevant affiliations or financial involvement with any organization or entity with a financial interest in or financial conflict with the subject matter or materials discussed in the manuscript. This includes employment, consultancies, honoraria, stock ownership or options, expert testimony, grants or patents received or pending, or royalties.

No writing assistance was utilized in the production of this manuscript.

\section{Bibliography}

1 Ehrlich P: The Collected Papers of Paul Ehrlich. Himmelweit F (Ed.). Pergamon, London, UK (1960).

2 Strebhardt K, Ullrich PAU: Ehrlich's magic bullet concept: 100 years of progress. Nat. Rev. Can. 8, 473-480 (2008).
3 Weiner LM, Surana R, Wang S: Monoclonal antibodies: versatile platforms for cancer immunotherapy. Nat. Rev. Immunol. 10, 317-327 (2010).

4 Campoli M, Ferris R, Ferrone S, Wang X: Immunotherapy of malignant disease with tumor antigen-specific monoclonal antibodies. Clin. Cancer Res. 16, 11-20 (2010).
5 Ross JS, Torres-Mora J, Wagle N, Jennings TA, Jones DM: Biomarker-based prediction of response to therapy for colorectal cancer: current perspective. Am. J. Clin. Pathol. 134, 478-490 (2010).

6 Saif MW: Colorectal cancer in review: the role of the EGFR pathway. Exp. Opin. Investig. Drugs 19, 357-369 (2010). 
7 Ferris RL, Jaffee EM, Ferrone S: Tumor antigen-targeted, monoclonal antibody based immunotherapy: clinical response, cellular immunity, and immunoescape. J. Clin. Oncol. 28, 1-10 (2010).

8 von Minckwitz G, du Bois A, Schmidt M et al.: Trastuzumab beyond progression in human epidermal growth factor receptor 2-positive advanced breast cancer: a German Breast Group 26/Breast International Group 03-05 study. J. Clin. Oncol. 27, 1999-2006 (2009).

9 Alfaro C, Suarez N, Gonzalez A et al.: Influence of bevacizumab, sunitinib and sorafenib as single agents or in combination on the inhibitory effects of VEGF on human dendritic cell differentiation from monocytes. Br. J. Cancer 100, 1111-1119 (2009).

10 Osada T, Chong G, Tansik R et al:: The effect of anti-VEGF therapy on immature myeloid cell and dendritic cells in cancer patients. Cancer Immunol. Immunother. 57, 1115-1124 (2008).
11 Wang SY, Weiner G: Complement and cellular cytotoxicity in antibody therapy of cancer. Expert Opin. Biol. Ther. 8, 759-768 (2008).

12 Abès R, Dutertre CA, Agnelli L, Teillaud JL: Activating and inhibitory $\mathrm{Fc} \gamma$ receptors in immunotherapy: being the actor or being the target. Expert Rev. Clin. Immunol. 5, 735-747 (2009).

13 Desjarlais JR, Lazar GA, Zhukovsky EA, Chu SY: Optimizing engagement of the immunesystem by antitumor antibodies: an engineer's perspective. Drug Discov. Today 12, 898-910 (2007).

14 Clynes R: Antitumor antibodies in the treatment of cancer: $F_{c}$ receptors link opsonic antibody with cellular immunity. Hematol. Oncol. Clin. North Am. 20, 585-612 (2006).

15 Kalergis AM, Ravetch JV: Inducing tumor immunity through the selective engagement of activating $\mathrm{F}_{\mathrm{c}} \gamma$ receptors on dendritic cells. J. Exp. Med. 195, 1653-1659 (2002).
16 Beum PV, Mack DA, Pawluczkowycz AW, Lindorfer MA, Taylor RP: Binding of rituximab, trastuzumab, cetuximab, or $\mathrm{mAb}$ T101 to cancer cells promotes trogocytosis mediated by THP-1 cells and monocytes. J. Immunol. 181, 8120-8132 (2008).

17 Galizia G, Lieto E, De Vita F et al.: Cetuximab, a chimeric human mouse anti-epidermal growth factor receptor monoclonal antibody, in the treatment of human colorectal cancer. Oncogene 26, 3654-3660 (2007).

18 Boruchov AM, Heller G, Veri MC, Bonvini E, Ravetch JV, Young JW: Activating and inhibitory $\operatorname{IgG} \mathrm{Fc}_{\mathrm{c}}$ receptors on human DCs mediate opposing functions. J. Clin. Invest 115, 2914-2923 (2005).

19 Zhang CY, Booth JW: Divergent intracellular sorting of Fc $\gamma$ RIIA and Fc $\gamma$ RIIB2. J. Biol. Chem 285(44), 34250-34258 (2010). 